\title{
Similarity of internal and external friction: Soft matter frictional instabilities obey mean field dissipation through slip avalanches
}

\author{
S. Zheng, ${ }^{1}$ J. M. Urueña, ${ }^{2}$ A. C. Dunn, ${ }^{3}$ J. T. Uhl, ${ }^{4, *}$ and K. A. Dahmen ${ }^{1, \dagger}$ \\ ${ }^{1}$ Department of Physics, University of Illinois at Urbana-Champaign, 1110 W. Green Street, Urbana, Illinois 61801, USA \\ ${ }^{2}$ Department of Mechanical and Aerospace Engineering, University of Florida, Gainesville, Florida 32611, USA \\ ${ }^{3}$ Department of Mechanical Science and Engineering, University of Illinois at Urbana-Champaign, Urbana, Illinois 61801, USA \\ ${ }^{4}$ Los Angeles, California, USA
}

(Received 6 June 2018; accepted 13 July 2020; published 20 October 2020)

\begin{abstract}
Resistance to slip across hydrogel surfaces is typically minimal, and sliding is smooth. However, recent surface friction experiments under high applied pressures caused stick-slip behavior between a glass probe and polyacrylamide hydrogel. In this paper we analyze the surface-based interface and its behavior similar to the more internal slip avalanches that occur in plastic deformations of metallic glasses and crystals using statistical descriptions. We find that the stick-slip surface friction satisfies universal power laws and scaling functions predicted by a simple mean field theory (MFT). We provide the rescaled average avalanche slip rate profiles, or average avalanche "shapes," defined as the average slip rate versus time, averaged over all avalanches with similar durations or sizes. We show that the avalanche shapes obtained in this friction experiment are consistent with MFT. This suggests the similar character of force buildup and release events in internal slip and surface friction for this unique data set, and the suitability of surface microfriction experiments to explore this space.
\end{abstract}

DOI: 10.1103/PhysRevResearch.2.042016

\section{INTRODUCTION}

Friction between two solid materials that are slowly being moved past each other can proceed in an intermittent fashion, with sudden slips or slip avalanches, separated by quiet times without any slipping activity. Here we compare the statistics and the dynamics of these slip events with the predictions of a simple mean field model that describes slips inside solid materials. The agreement between experiments and theory links surface friction to internal friction and thereby leads to a predictive understanding that can be used to guide the design of better materials.

In general, slip avalanches locally dissipate the energy at an interface between larger bodies; this can occur on large scales such as earthquakes [1] down to nanoscale dislocation motion in crystalline solids [2]. In nanocrystals, for example, the slips typically occur exceedingly quickly, making it challenging to measure them experimentally. If they occur inside the solid, the buried interface often forces the measurement to be located far from the active regions and the resolution of time and force are not easily captured [3].

In contrast, surface friction studies can provide more access to slip behavior and specifically stick-slip motions be-

\footnotetext{
*Retired.

$\dagger$ dahmen@illinois.edu
}

Published by the American Physical Society under the terms of the Creative Commons Attribution 4.0 International license. Further distribution of this work must maintain attribution to the author(s) and the published article's title, journal citation, and DOI. cause the samples are often larger, they are sometimes transparent, and the slips proceed more slowly. The experiments can then elicit the behavior based on the surface composition and topography, applied normal force, and driving translation speed or displacement [4]. The friction experiments also provide information about material changes. For example, plastic deformation manifests in the stick-slip behavior via shear weakening, shear strengthening, or approximately zero weakening [5-7]. Rate-dependent surface deformation is a wellestablished field, but still the durations of individual slips, caused by elastic (depinning) deformation or plastic deformation, can be small, on the order of nanoseconds to microseconds [8]. We discuss a simple statistical mean field model that can accurately describe these effects and compare the predictions with sufficient experimental data for validation.

According to rubber elasticity, the material recoil of soft materials such as elastomers, during slip, is slower than for hard materials [9]. This slowed-down dynamics of soft materials provides us with dramatically increased temporal resolution of the individual slip events. Recently, a material even softer than an elastomer, a hydrogel of $E^{\prime} \sim 10 \mathrm{~s} \mathrm{kPa}$, experienced stick-slip motion in a microtribological measurement while sliding under a smooth glass probe. This extends the slip timescale up to milliseconds. In addition, the compliant surface provides a conformal contact and the area of contact can spread to the entire interface. In contrast, in hard materials the interfaces touch only at the asperity peaks.

The dramatically slowed-down dynamics in soft materials overcomes the experimental challenges of time resolution and isolated nanoscopic contact areas. Also, in soft materials such as hydrogels we can safely exclude roughness, electronic properties, or crystallographic properties as the origins of the 
stick-slip behavior, thereby drastically simplifying the data analysis.

Hydrogels are typically known for smooth sliding, or excellent lubricity, and as such are used for low-friction surfaces in biomedicine (catheter coatings, contact lenses, and drug delivery [10]) and industrial applications (drilling muds [11]). However, when a surface hydrogel layer with high water content (greater than $80 \%$ water by mass) is compressed under pressures approaching its elastic modulus and then subjected to prescribed shear, the collapsed layer results in frictional instability and gross stick-slip events [12]. In this work we induce stick-slip behavior under a variety of pressures, analyze the behavior, and compare the results to a simple model to understand the behavior.

Specifically, we analyze the friction force avalanches resulting from shear of a glass-hydrogel interface under seven different normal loads. We characterize the force drop amplitudes in the force versus time data sets. We show that the stick-slip behaviors of the friction between a hydrogel surface and a glass probe satisfy universal power laws and scaling functions predicted by a simple mean field theory for slip avalanches inside solid materials [6]. The statistics and dynamics of the slip events during surface friction agree with those of the slips occurring during internal friction inside the bulk of many solid materials, as was predicted by the same mean field model [5-7].

\section{METHODS}

The simple mean field theory (MFT) [6] provides a unified understanding of plasticity for many materials, such as crystals, metallic glasses, and densely packed granular solids [7]. The model assumes that the vast majority of solid materials have weak spots. It assumes that the weak spots slip whenever the local force exceeds some threshold. For our friction experiments this implies that weak spots at the frictional interface slip whenever the applied force from the cantilever lateral deflection exceeds the static friction coefficient in the contact. The weak spot undergoes a local slip until the stress in the contact is reduced to some local (random) arrest stress. A slipping weak spot can trigger other elastically coupled weak spots to slip by increasing the stress at these points. This process creates an avalanche of slip instabilities. For brittle materials, the model assumes that the threshold to slipping can be weakened after a slip similar to static versus dynamic friction. In that case it predicts two types of slip avalanches: (1) small slip avalanches that have self-similar dynamics and (2) large slip avalanches that have cracklike scaling behaviors and recur almost periodically at constant imposed average displacement rate. The simple MFT predicts both the power-law exponents and scaling function of the slip-size distributions. The mean field approximation has been proven mathematically to apply when the slip is localized to a plane, as is the case in friction experiments on a plane (like here) and in materials with shear planes such as bulk metallic glasses and earthquakes [6,13].

To elicit systematic and detailed stick-slip behavior and to test the model predictions, we measure the friction forces between a thin hydrogel layer and a hemispherical broad-radius borosilicate glass probe under different applied normal forces. The thin hydrogel layer in this study is
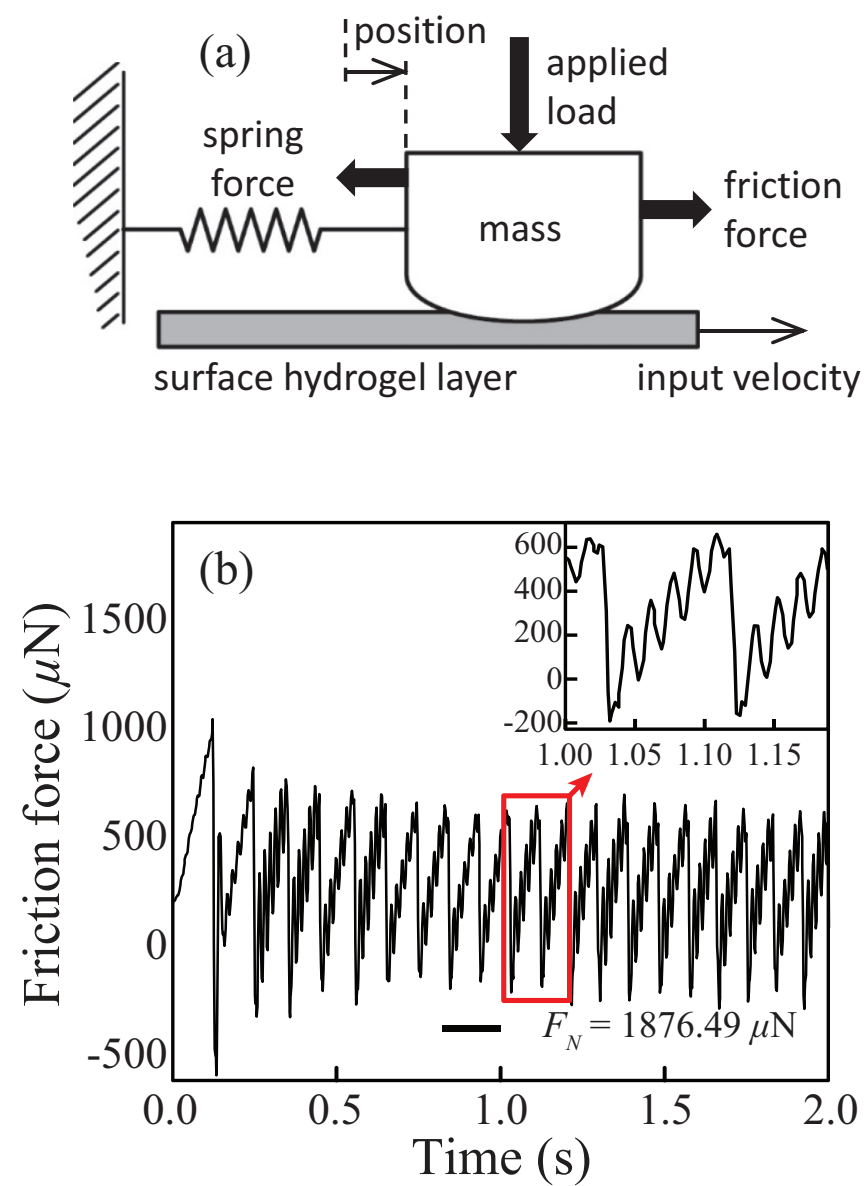

FIG. 1. (a) Schematic of the experimental setup including a smooth glass frictional probe, surface hydrogel, and force measurement mechanism through static flexure deflection. (b) Example force drops versus time for the total sliding motion. The normal force $F_{N}$ in this example is $1876.49 \mu \mathrm{N}$. The inset shows a close-up version, which allows us to resolve small force drops.

approximately $5 \mu \mathrm{m}$ thick with an effective elastic modulus of $25 \mathrm{kPa}$ as measured by contacting, colloidal probe atomic force microscopy. The surface mechanics of the material under low pressure were characterized in prior work [12]. In a custom microtribometer, a glass probe of radius $3 \mathrm{~mm}$ is loaded against the thin surface hydrogel layer up to a normal load of $F_{N}=2000 \mu \mathrm{N}$, all submerged in a buffered saline solution. Then the hydrogel is translated at an input velocity of $V=200 \mu \mathrm{m} / \mathrm{s}$ by a high-resolution linear piezoelectric stage (PI USA, Auburn, MA) to a stroke length of $s=600 \mu \mathrm{m}$ [Fig. 1(a)]. The stage slides back and forth for multiple cycles and the normal force is decreased to incrementally lower cycle-averaged values ranging from $F_{N}=1876 \mu \mathrm{N}$ down to $F_{N}=127 \mu \mathrm{N}$. Based on a bed-of-springs thin-film elasticity model $[14,15]$, those applied forces are anticipated to result in exceedingly high pressures of $P=25 \mathrm{kPa}$ down to $P=8 \mathrm{kPa}$, which means the surface is completely compressed (one-dimensional strain through the surface layer thickness $\left.\varepsilon_{V} \rightarrow 1\right)$ at the higher pressures and still experiences $\varepsilon_{V} \sim 0.3$ at the lowest pressure. During the translation, lateral forces (or friction forces) are recorded at $544 \mathrm{~Hz}$ through analog voltages using a data acquisition board and custom software (National Instruments, Austin, TX). The force is 

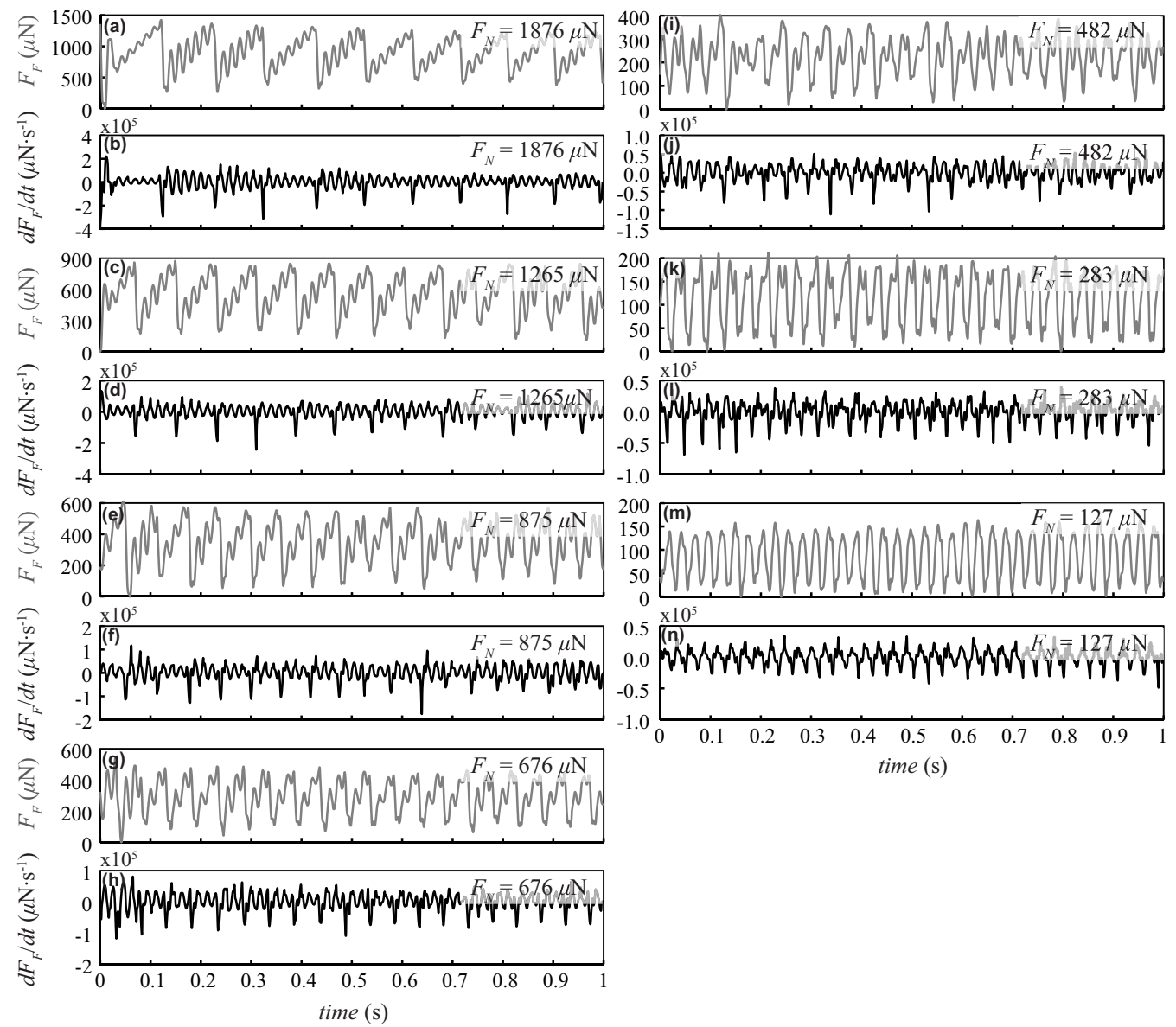

FIG. 2. Pairs of plots juxtaposing the measured friction force magnitudes $F_{F}$ (gray data) and calculated change in force with time $d F_{F} / d t$ (black data) over the course of $1 \mathrm{~s}$. Plots are organized according to decreasing levels of applied normal force for (a) and (b) $F_{N}=1876 \mu \mathrm{N}$, (c) and (d) $F_{N}=1265 \mu \mathrm{N}$, (e) and (f) $F_{N}=875 \mu \mathrm{N}$, (g) and (h) $F_{N}=676 \mu \mathrm{N}$, (i) and (j) $F_{N}=482 \mu \mathrm{N},(\mathrm{k})$ and (l) $F_{N}=283 \mu \mathrm{N}$, and (m) and (n) $F_{N}=283 \mu \mathrm{N}$. The near periodicity of the large force drops in the data reflects a frictional weakening mechanism, related to static versus dynamic friction, that produces almost-periodic large slips in addition to smaller slips in between [6,17-19].

calculated through the deflection of a lateral spring of stiffness $k=87.46 \mathrm{~N} / \mathrm{m}$, read by a capacitive probe (Lion Precision, St. Paul, MN). Data quality was ensured by reducing mechanical and electrical noise through the use of vibration isolation beneath the instrument, as well as high-resolution capacitive sensors and adequate analog voltage sensitivity. The noise is estimated to be in the single- $\mu \mathrm{N}$ range, which is smaller than the magnitudes of the measured force drops.

In the data analysis, we numerically differentiated the friction time series $F(t)$ to obtain $V(t)=\frac{-d[F(t)]}{d t}$ (see the Supplemental Material for details [16]). The interval over which a force drop has occurred is defined by $V\left(t_{\text {start }}\right)=$ $V\left(t_{\text {end }}\right)=0$ and $V(t)>0$ for $t \in\left(t_{\text {start }}, t_{\text {end }}\right)$, where $t_{\text {start }}$ and $t_{\text {end }}$ are the starting and ending times of the avalanche. Therefore, the duration of the avalanche is $t_{\text {end }}-t_{\text {start }}$ and the size of the avalanche is $S \equiv-\left[F\left(t_{\text {end }}\right)-F\left(t_{\text {start }}\right)\right]$. In the following we use the symbol $F$ to denote the force drop size $S$ of an avalanche, i.e., $F \equiv S$.

\section{RESULTS}

For this particular hydrogel surface, it was proposed that high applied pressures collapse the compliant hydrogel layer and force water squeezeout in a pressure-driven Darcy flow [12]. The drastically compressed material prevents the interface from slipping easily and converts it to one that responds in an unstable way to lateral force buildup. As the force deflecting the spring exceeds the static friction force of the interface under a constant input velocity, a cascade of slip events initiates. Friction forces over a representative duration of $1 \mathrm{~s}$ are shown in Fig. 2. For a magnitude of applied normal force of $F_{N}=1876 \mu \mathrm{N}$, the associated frictional forces go intermittently between zero and $\sim 800 \mu \mathrm{N}$, which presents unstable instantaneous friction coefficients ranging from a negligibly small number up to $\mu \sim 0.43$, a high number for a typically lubricious surface. The near periodicity of the large force drops in the data reflects a frictional weakening mechanism, related to static versus dynamic friction, that produces almost-periodic large slips in addition to smaller slips in between [6,17-19].

For the highest applied normal load of $F_{N}=1876 \mu \mathrm{N}$, we found 385 slip events in total. The sizes of the slip events range from $F=0.567$ to $1025.5 \mu \mathrm{N}$ and the durations $T$ range from $T=0.3$ to $12.5 \mathrm{~ms}$. Data for every normal load can be found in the Supplemental Material [16]. No filtering was necessary due to the experimental capabilities. Here we 

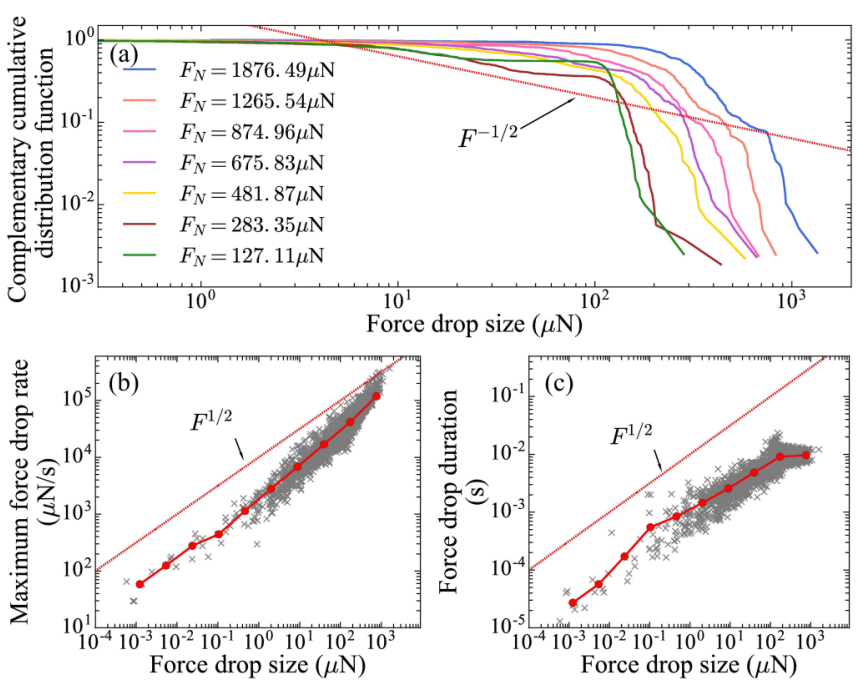

FIG. 3. (a) Cumulative distribution of friction force drops during slip events at interfaces nominally loaded to normal forces between $F_{N}=127.11$ and $1876.49 \mu \mathrm{N}$. The red dashed line has a slope of $1 / 2$, which is predicted by a simple mean field model. Measurements are expected to be valid for force drops $F>1 \mu \mathrm{N}$ based on the sensitivity of the data acquisition system. (b) Maximum force drop rate versus force drop size and (c) force drop duration versus force drop size. In both figures, there are 3022 data points in total and the normal forces range from $F_{N}=127.11$ to $1876.49 \mu \mathrm{N}$. The slopes of the red dashed lines again indicate the corresponding scaling exponents predicted by the same mean field model. The slopes of the red lines are $1 / 2$. Data points are split into ten bins, each of which has the same number of data points. Red dots are average values of each bin. At the largest force drop sizes $F>120 \mu \mathrm{N}$, the force drop duration no longer depends on force drop size (i.e., no durations are above $T>0.02 s$ ).

briefly summarize some of the model predictions for the force drop statistics between a hydrogel surface and a glass probe: The complementary cumulative size distribution $C(F)$ scales as $C(F) \sim F^{-1 / 2}$ for force drops in the power-law scaling regime [Fig. 3(a)] for most of the normal forces that were employed (except for the highest normal forces, where the scaling regime has shrunk too much to be visible in the figure). The large events seen especially for the highest forces are reflecting a weakening effect, such as static versus dynamic friction which is predicted to create large slips of similar size in addition to the smaller ones [5-7,17-19], consistent with the experiments shown here. Moreover, for the events in the scaling regime the maximum force drop rate $v_{m}$ and force drop duration $T$ at size $F$, scale as $v_{m} \mid F \sim(F)^{\sigma \rho} \sim(F)^{1 / 2}$ and $T \mid F \sim F^{\sigma \nu z} \sim F^{1 / 2}$, respectively, which are in good agreement with the model predictions [Figs. 3(b) and 3(c)]. Here $\sigma \rho$ and $\sigma v z$ are critical exponents that are predicted by the model to be universal, i.e., determined by basic properties such as symmetries, dimensions, and range of interactions. They are independent of the microscopic details of the system $[5,6]$. The names of these exponents are complicated for historical reasons. The mean field model predicts $\sigma \rho=1 / 2$ and $\sigma v z=1 / 2[5,6,20]$. The scaling for the largest events again is slightly different from that of the small ones, consistent with the weakening prediction of the model that predicts

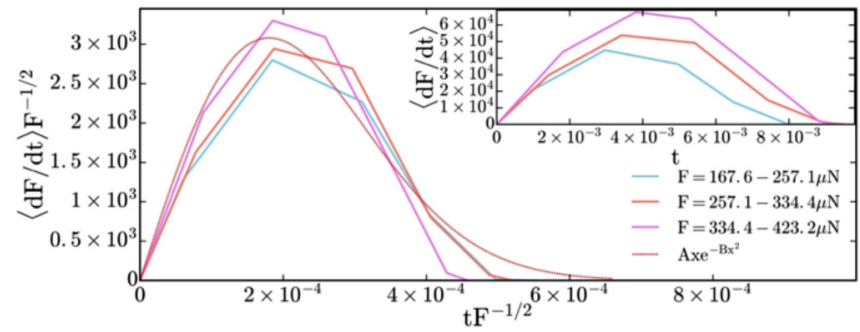

FIG. 4. Average force drop rate versus time for different force drop sizes $F$ (scaling both axes by a factor of $F^{-1 / 2}$ ). Profiles are averaged over force drops that have similar force drop sizes. The inset shows the unscaled average force drop rate versus time. The predicted collapse function represented by the red dashed line has the form $A x e^{-B x^{2}}$, where $A$ and $B$ are nonuniversal constants $A=$ $2.82 \times 10^{7}$ and $B=1.54 \times 10^{7}$. The collapse shows that the model predictions for the scaling exponents and the scaling function are consistent with the experimental data.

that weakening correspondingly changes the dynamics for the largest events [5-7,17-19].

Figure 4 shows the average temporal profiles of force drops of given size in the scaling regime of $C(F)$. Here the average temporal profile is defined as the force drop rate as a function of time averaged over all force drops of similar size $F$. After scaling both axes by $F^{-1 / 2}$, profiles collapse onto the predicted collapse function, represented by the red dashed line. Similarly, the temporal profiles averaged over force drops of similar durations $T$ also agrees with the model predictions when both axes are rescaled by their maxima (Fig. 5). The result shows that the average shape is an inverted parabola. Similar results for a smaller normal load are shown in the Supplemental Material [16].

The collapses shown in Figs. 4 and 5 and in [16] are consistent with the predictions of the simple mean field model $[5,6]$. The model predicts that the small avalanches within the power-law scaling regime should have average temporal profiles that are simply rescaled versions of the larger avalanches in the scaling regime, reflecting the self-similarity

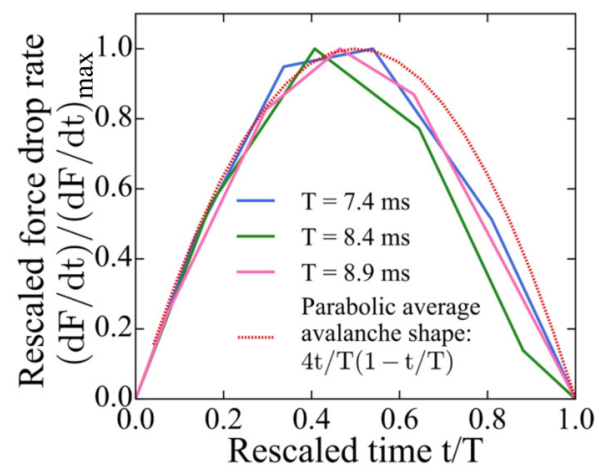

FIG. 5. Rescaled force drop rate versus rescaled time (both axes are scaled by their maxima). Profiles are averaged over force drops that have similar durations. The red dashed line shows the model prediction of a parabolic average avalanche shape, which is very similar to the experimental data. The slight asymmetry to the left may reflect frictional delay effects, similar to those in earthquakes [21]. 
in the dynamics that is expected near a critical point. The model predicts not just the rescaling exponents but also the scaling functions of these average profiles. The predicted scaling functions agree with the data as shown in Figs. 4 and 5 and in [16].

Specifically, the model predicts that the force drop rate $\langle d F / d t(t \mid T)\rangle$ averaged over all avalanches of given duration $T$ in the scaling regime should scale as a parabola: $\langle d F / d t(t \mid T)\rangle / T \sim[1-(t / T)](t / T)$. Similarly, the force drop rate $\langle d F / d t(t \mid F)\rangle$ averaged over all force drops of size $F$ should scale as $\langle d F / d t(t \mid F)\rangle / F^{1 / 2} \sim$ $\left(t / F^{1 / 2}\right) \exp \left[-B\left(t / F^{1 / 2}\right)^{2}\right]$, where $B$ is the only fitting constant. Both of these predicted profiles $[5,6]$ are consistent with the experimental data in Figs. 4 and 5.

\section{DISCUSSION}

The described mean field model appears to accurately describe the stick-slip motion observed for a glass-hydrogel interface under high pressures from $40 \%$ to $100 \%$ of the Young's modulus of the surface hydrogel layer. The periodic large slips interleaved with irregular small slips indicate that the interface can be described as shear weakening; similar to static versus kinetic Coulomb friction, regions that have slipped once during an avalanche will slip again at much lower forces than was originally needed to start the first slip in a slip avalanche.

Thorough investigations of soft surface stick-slip mechanisms have determined that they arise from synergistic cooperation of mechanisms such as viscoelastic deformation, adhesion, and even surface damage [22-28]. While some hydrogels can exhibit viscoelastic behavior, the parabolic symmetry of the normalized average force drop rate for slips of given total duration $T$ (Fig. 5) indicates that inertial effects such as viscoelastic recoil can be neglected, at least for the smaller slips within the avalanche [5,7]. Further, surface deformation was expected to remain in the plane of contact due to the high rotational stiffness of the probe flexure [22], preventing out-of-plane surface displacements, which would complicate the stresses in the interface (observed in elastomeric stickslip motion [29]). In addition, no surface damage was observed, and surface energies in a buffered saline solution are negligible.

Since those mechanics are not expected to contribute significantly, other characteristics specific to the hydrogel surface layer, such as the porous nature of the surface, may contribute to the observed stick-slip behavior. Prior work postulates that high applied pressures collapse the surface hydrogel layer [12] and recent poroelastic modeling of hydrogel lubrication shows that fluid squeezeout alters the surface properties under slip [30]. The low modulus of elasticity of this material ( $\sim 25 \mathrm{kPa}$ ) would make it highly susceptible to fluid squeezeout under the pressures of these measurements, estimated to be between 8 and $25 \mathrm{kPa}$. The alterations to the hydrogel surface under high pressures, perhaps such as compaction, manifest as unstable friction behavior that is well described by the shear-weakening slip avalanche mean field model.

The experiments described in this work load two very dissimilar surfaces (a smooth glass against a very compliant hydrogel surface) and prescribe a driving speed in the far field, eliciting observable stick-slip events in the form of force drops along the slip direction. The statistical similarity of the slip statistics and dynamics to those of internal interfaces observed in the deformation of bulk metallic glasses [2] supports the model prediction that surface friction and internal friction share similar energy-dissipation characteristics [31]. This similarity is also reminiscent of the scalability of brittle fracture mechanics between hard materials and soft materials [32]. Future work is needed to define the limits of similarity and the subtleties of material properties. Instrumented surface friction measurements in the form of microtribometry show promise as a technique for systematically varying the surfaces used to compose the interface (material properties and surface energies), along with the prescribed loading conditions and driving speeds. Connections to models and friction measurements varying other experimental tuning parameters such as driving speeds are left for future work [22,29,33].

\section{CONCLUSION}

This study has three main results. (1) Microfriction experiments at high pressures were performed on hydrogel surface layers to induce stick-slip behaviors. The force drop events were observed when applied pressures approached the effective elastic modulus of the surface hydrogel layer. (2) The scaling behavior of the maximum force drop rate as a function of force drop size and the force drop duration as a function of force drop size follow power laws that agree with predictions of a simple mean field model. (3) The force drop size distributions and temporal shapes of avalanches are consistent with the predictions from MFT, suggesting that the dynamics at interfaces created by the surfaces of dissimilar materials and within shear bands or interfaces internal to a material have the same statistical scaling behavior [17]. In addition, instrumented surface friction experiments show promise as a way to systematically explore these similarities.

\section{ACKNOWLEDGMENTS}

We thank the University of Florida Soft Matter Engineering group for instrument use. We thank Will McFaul, Alan Long, and Mohammed Sheikh for helpful conversations. We thank the National Science Foundation for support through Grant No. NSF CBET 1336634 (K.A.D.) and CMMI 1751945 (A.C.D.).
[1] P. Bak and C. Tang, J. Geophys. Res. 94, 15635 (1989).

[2] R. Maass, M. Wraith, J. T. Uhl, J. R. Greer, and K. A. Dahmen, Phys. Rev. E 91, 042403 (2015) .

[3] W. G. Sawyer and K. J. Wahl, MRS Bull. 33, 1145 (2008).
[4] I. Hutchings, Tribology, Friction and Wear of Engineering Materials, 1st ed. (Butterworth-Heinemann, Oxford, 1992).

[5] K. A. Dahmen, Y. Ben-Zion, and J. T. Uhl, Nat. Phys. 7, 554 (2011). 
[6] K. A. Dahmen, Y. Ben-Zion, and J. T. Uhl, Phys. Rev. Lett. 102, 175501 (2009).

[7] J. T. Uhl, S. Pathak, D. Schorlemmer, X. Liu, R. Swindeman, B. A. W. Brinkman, M. LeBlanc, G. Tsekenis, N. Friedman, R. Behringer, D. Denisov, P. Schall, X. Gu, W. J. Wright, T. Hufnagel, A. Jennings, J. R. Greer, P. K. Liaw, T. Becker, G. Dresen, and K. A. Dahmen, Sci. Rep. 5, 16493 (2015).

[8] F. F. Abraham, R. Walkup, H. Gao, M. Duchaineau, T. Diaz De La Rubia, and M. Seager, Proc. Natl. Acad. Sci. USA 99, 5783 (2002).

[9] K. Viswanathan, N. K. Sundaram, and S. Chandrasekar, Soft Matter 12, 5265 (2016).

[10] C. M. Kirschner and K. S. Anseth, Acta Mater. 61, 931 (2013).

[11] R. Zolfaghari, A. A. Katbab, J. Nabavizadeh, R. Y. Tabasi, and M. H. Nejad, J. Appl. Polym. Sci. 100, 2096 (2006).

[12] A. C. Dunn, J. M. Urueña, Y. Huo, S. S. Perry, T. E. Angelini, and W. G. Sawyer, Tribol. Lett. 49, 371 (2013).

[13] D. S. Fisher, K. A. Dahmen, S. Ramanathan, and Y. Ben-Zion, Phys. Rev. Lett. 78, 4885 (1997).

[14] A. C. Rennie, P. L. Dickrell, and W. G. Sawyer, Tribol. Lett. 18, 499 (2005).

[15] P. Põdra and S. Andersson, Wear 207, 79 (1997).

[16] See Supplemental Material at http://link.aps.org/supplemental/ 10.1103/PhysRevResearch.2.042016 for details.

[17] J. Antonaglia, W. J. Wright, X. Gu, R. R. Byer, T. C. Hufnagel, M. LeBlanc, J. T. Uhl, and K. A. Dahmen, Phys. Rev. Lett. 112, 155501 (2014).
[18] A. A. Long, D. V. Denisov, P. Schall, T. C. Hufnagel, X. Gu, W. J. Wright, and K. A. Dahmen, Granul. Matter 21, 99 (2019).

[19] D. V. Denisov, K. A. Lorincz, J. T. Uhl, K. A. Dahmen, and P. Schall, Nat. Commun. 7, 10641 (2016).

[20] M. LeBlanc, A. Nawano, W. J. Wright, X. Gu, J. T. Uhl, and K. A. Dahmen, Phys. Rev. E 94, 052135 (2016).

[21] A. P. Mehta, K. A. Dahmen, and Y. Ben-Zion, Phys. Rev. E 73, 056104 (2006).

[22] T. Baumberger and C. Caroli, Adv. Phys. 55, 279 (2006).

[23] J. H. Dieterich, Pure Appl. Geophys. 116, 790 (1978).

[24] F. Heslot, T. Baumberger, B. Perrin, B. Caroli, and C. Caroli, Phys. Rev. E 49, 4973 (1994).

[25] S. Maegawa, F. Itoigawa, and T. Nakamura, Tribol. Int. 96, 23 (2016).

[26] C. J. Rand and A. J. Crosby, Appl. Phys. Lett. 89, 261907 (2006).

[27] Y. Kligerman and M. Varenberg, Tribol. Lett. 53, 395 (2014).

[28] S. Maegawa and K. Nakano, Wear 268, 924 (2010).

[29] M. K. Chaudhury and K. H. Kim, Eur. Phys. J. E 23, 175 (2007).

[30] E. R. Reale and A. C. Dunn, Soft Matter 13, 428 (2017).

[31] M. Paczuski and S. Boettcher, Phys. Rev. Lett. 77, 111 (1996).

[32] T. Goldman Boué, R. Harpaz, J. Fineberg, and E. Bouchbinder, Soft Matter 11, 3812 (2015).

[33] T. Yamaguchi, M. Morishita, M. Doi, T. Hori, H. Sakaguchi, and J. P. Ampuero, J. Geophys. Res. 116, B12306 (2011). 